\title{
SCIENTIFIC REPORTS

\section{OPEN Peripheral Blood Mononuclear Cell Oxytocin and Vasopressin Receptor Expression Positively Correlates \\ with Social and Behavioral Function in Children with Autism}

Received: 13 June 2019

Accepted: 28 August 2019

Published online: 17 September 2019

\author{
Irena Voinsky ${ }^{1}{ }^{1}$, Sirish C. Bennuri ${ }^{2}$, Julie Svigals ${ }^{1}$, Richard E. Frye $\mathbb{D}^{3,4}$, Shannon Rose ${ }^{2}{ }^{2} \&$ \\ David Gurwitz (iD ${ }^{1,5}$
}

The peptide hormone oxytocin is an established regulator of social function in mammals, and dysregulated oxytocin signaling is implicated in autism spectrum disorder (ASD). Several clinical trials examining the effects of intranasal oxytocin for improving social and behavioral function in ASD have had mixed or inclusive outcomes. The heterogeneity in clinical trials outcomes may reflect large interindividual expression variations of the oxytocin and/or vasopressin receptor genes OXTR and AVPR1A, respectively. To explore this hypothesis we examined the expression of both genes in peripheral blood mononuclear cells (PBMC) from ASD children, their non-ASD siblings, and age-matched neurotypical children aged 3 to 16 years of age as well as datamined published ASD datasets. Both genes were found to have large inter-individual variations. Higher OXTR and AVPR1A expression was associated with lower Aberrant Behavior Checklist (ABC) scores. OXTR expression was associated with less severe behavior and higher adaptive behavior on additional standardized measures. Combining the sum expression levels OXTR, AVPR1A, and IGF1 yielded the strongest correlation with ABC scores. We propose that future clinical trials in ASD children with oxytocin, oxytocin mimetics and additional tentative therapeutics should assess the prognostic value of their PBMC mRNA expression of OXTR, AVPRIA, and IGF1.

The peptide hormone oxytocin is strongly implicated in social behavior and is conserved in mammals. Oxytocin has been extensively studied in the context of social function in animals and humans, in particular in children and adults with autism spectrum disorder $(\mathrm{ASD})^{1-3}$. Intranasal oxytocin was shown to improve social function and empathy in healthy individuals ${ }^{4-6}$, and is thus a candidate therapeutic for children with ASD.

It has been suggested that genetic variations of the OXTR alleles, promoter methylation, and/or expression levels in brain areas implicated in social function may contribute to the variable response observed in clinical trials with intranasal oxytocin in $\mathrm{ASD}^{7,8}$. In addition, differences between and within animal species in sociality may in part be explained by substantial differences in OXTR expression levels within the social brain network, such as the amygdala, the anterior cingulate cortex, the prefrontal cortex and the nucleus accumbens ${ }^{9}$. In primates, OXTR is also expressed in the superior colliculus, the pulvinar, and the primary visual cortex, where oxytocin functions as a modulator of visual processing and allocation of attention ${ }^{10}$. Thus, variations in OXTR expression seem critical for the diversity of social behaviors across and within mammal and in particular primate species, including humans. Indeed, inter-individual variations in OXTR expression levels were shown to be associated with resilience to the effects of neonatal isolation on adult social attachment in female prairie voles ${ }^{11}$.

${ }^{1}$ Department of Human Molecular Genetics and Biochemistry, Sackler Faculty of Medicine, Tel Aviv University, Tel Aviv, Israel. 'Department of Pediatrics, University of Arkansas for Medical Sciences and Arkansas Children's Research Institute, Little Rock, AR, USA. ${ }^{3}$ Department of Child Health, University of Arizona College of Medicine-Phoenix, Phoenix, AZ, USA. "Barrow Neurological Institute at Phoenix Children's Hospital, Phoenix, AZ, USA. ${ }^{5}$ Sagol School of Neuroscience, Tel Aviv University, Tel Aviv, Israel. Correspondence and requests for materials should be addressed to R.E.F. (email: rfrye@phoenixchildrens.com)orS.R. (email:SROSE@uams.edu) or D.G. (email: gurwitz@post.tau.ac.il) 
Several clinical trials have studied intranasal oxytocin as a tentative ASD therapeutic for improving social and behavioral function in children, adolescents, or adults diagnosed with ASD, albeit with mixed outcomes ${ }^{12-22}$. A follow-up study ${ }^{23}$ of one of these published clinical trials ${ }^{12}$ reported correlations of treatment efficacy with the OXTR single nucleotide polymorphisms (SNPs) rs53576 and rs2254298; another oxytocin trial reported efficacy correlations with OXTR rs6791619 ${ }^{13}$. The large variation in these clinical trials could in-part reflect genetic or epigenetic variations in the expression levels of the oxytocin receptor gene, OXTR. In addition to oxytocin, the closely similar peptide hormone arginine-vasopressin is also implicated in social function via its V1AR receptor, encoded by AVPR1A ${ }^{24-27}$. Another peptide hormone studied as a tentative ASD therapeutic is insulin-like growth factor 1 (IGF-1), encoded in humans by IGF1. A single small study with recombinant human IGF-1 reported favorable response in ASD children ${ }^{28}$ and another study (NCT01970345) is ongoing.

We hypothesize that variations in OXTR, AVPR1A and IGF1 genes could explain the variation in response to these therapeutic agents in clinical trials. To explore this hypothesis we examined OXTR, AVPR1A and IGF1 expression levels in peripheral blood mononuclear cells (PBMC) samples from 63 ASD children, their non-ASD siblings, and matched neurotypical children aged 3-16 years (Table 1). We explore gene expression because we believe that expression levels, rather than SNPs, may afford a more robust precision medicine tool for ASD patients, as gene expression levels, unlike SNPs, also reflect epigenomic effects on transcription ${ }^{29,30}$. To supplement our gene expression data, we applied datamining of published Gene Expression Omnibus (GEO) datasets for exploring OXTR and AVPR1A expression variations in blood and postmortem brain samples from ASD and matched controls, respectively. Our findings suggest that PBMC expression levels of OXTR, AVPR1A and IGF1 correlate with several standardized measures of behavior and development, including the Aberrant Behavior Checklist (ABC), Vineland Adaptive Behavior Scale (VABS), Social Responsiveness Scale (SRS), and Childhood Behavior Checklist (CBCL). Based on our findings we suggest that the PBMC expression levels of these genes should be evaluated as tentative prognostic biomarkers by clinical trials with oxytocin or other tentative ASD therapeutics.

\section{Results}

The age, sex, and key ASD scores of study participants are presented in Table 1. As both oxytocin and vasopressin are implicated in $\mathrm{ASD}^{1-3,24,25}$, we searched for expression differences of the genes which code for their receptors, OXTR and AVPR1A, in PBMCs collected from the study participants. No statistically significant differences were found for the PBMC mRNA expression levels of OXTR or AVPR1A between ASD children, their neurotypical siblings, and/or neurotypical age-matched children (Fig. 1a,b). Data mining of GEO datasets derived from ASD and control PBMC (GSE111176, GSE25507) or whole blood (GSE18123, GSE89594) confirmed the lack of differences in OXTR or AVPR1A expression levels in PBMC or whole blood from ASD and neurotypical controls (Fig. 1c-j).

Next, we searched for correlations between PBMC expression levels of OXTR and AVPR1A and the available social and behavioral scores of our study participants (see Methods; scores were recorded at the same time as blood sample collection for PBMC separation). We found that higher OXTR expression levels correlated with i) better development as measured by the VABS Adaptive behavioral composite, ii) less severe social impairment as measured by the SRS total t-score and iii) less severe behavior problems as measured by the lower total raw $\mathrm{ABC}$ scores and lower total CBCL t-scores (Fig. 2a-d). The PBMC expression of AVPR1A correlated with the participants total raw ABC scores, but not with their VABS, SRS, or CBCL scores (Fig. 2e; Supplementary Fig. 1e-g). Notably, no correlations with standardized scores were observed for the PBMC expression of CD38 (Supplementary Fig. 1a-d), a gene that codes for an enzyme that synthesizes and hydrolyzes cyclic adenosine $5^{\prime}$-diphosphate-ribose and taking part in oxytocin secretion ${ }^{31}$. In addition, we also observed a correlation of the participants total raw $\mathrm{ABC}$ scores and their expression levels of IGF1, coding for insulin-like growth factor 1 (Fig. 2g).

Since combining the expression levels of several genes may afford more robust diagnostic or prognostic power $^{32-36}$ and as the ABC scores of the ASD children correlated with the OXTR, AVPR1A, and IGF1 expression levels, we determined whether combining the expression of these genes affords a better correlation with ABC scores as compared to correlation with individual gene expression. As shown in Fig. $2 \mathrm{~h}$ the combined expression levels of all three genes yielded improved Pearson correlations (larger $r$ values and smaller $\mathrm{p}$ values) compared to each gene separately or with two-gene combinations(Fig. 2f). In order to verify the robustness of the correlation between the summed $O X T R, A V P R 1 A$, and IGF1 expression levels with ABC scores, we randomly selected 30 individuals from our dataset. For each of 500 random selections we determined the Pearson's correlation coefficient and p-value of the correlation between $\mathrm{ABC}$ score and the summed gene expression. We found that 410 $(82 \%)$ of these random subsets yielded a significant correlation ( $\mathrm{p}$-value $<0.05$ ) between $\mathrm{ABC}$ score and summed gene expression (mean Spearman's correlation coefficient: 0.47 ). This is compared to 25 (5\%) significant subsets expected by chance. This result demonstrates that the observed correlation is robust and is not underlined by specific outlier individuals.

\section{Discussion}

Dysfunctional signaling by the peptide hormones oxytocin and vasopressin were suggested as contributing to ASD. While the role of these hormones has been extensively studied in several mouse models of ASD 37,38 , and oxytocin receptor knockout mice display behavioral deficits resembling autism-related behaviors ${ }^{39}$, such mouse models typically reflect a single mutation or deletion, and do not reflect the real-world state and wide spectrum of ASD individuals. Our study aimed to explore correlations between development and behavior in children with ASD and PBMC expression levels of the oxytocin and vasopressin receptor genes OXTR or AVPR1A, respectively.

Our findings indicate that the severity of developmental and behavioral deficits in children with ASD aged 3 to 16 years is associated with their PBMC mRNA expression levels of OXTR (ABC, VABS, SRS, and CBCL scores), as well as with AVPR1A and IGF1 (ABC score). The PBMC expression of all three genes therefore seem to be associated with the severity of aberrant behavior in children with ASD. 


\begin{tabular}{|c|c|c|c|c|c|c|}
\hline Code & Age & Sex & VABS Social & SRS Total & ABC Total & CBCL Total \\
\hline \multicolumn{7}{|c|}{ Controls } \\
\hline $\mathrm{C} 001$ & 6.42 & M & 126 & $\mathrm{NC}$ & $\mathrm{NC}$ & $\mathrm{NC}$ \\
\hline $\mathrm{C} 002$ & 2.66 & $\mathrm{~F}$ & $\mathrm{NC}$ & $\mathrm{NC}$ & $\mathrm{NC}$ & $\mathrm{NC}$ \\
\hline $\mathrm{C} 003$ & 4.71 & $\mathrm{~F}$ & NC & NC & NC & $\mathrm{NC}$ \\
\hline $\mathrm{C004}$ & 6.49 & M & 103 & 46 & 22 & 51 \\
\hline $\mathrm{C} 005$ & 6.89 & $\mathrm{~F}$ & 114 & 82 & 59 & 75 \\
\hline C006 & 7.38 & F & 132 & NC & NC & NC \\
\hline $\mathrm{C} 007$ & 7.45 & M & 127 & NC & NC & $\mathrm{NC}$ \\
\hline $\mathrm{C} 008$ & 7.9 & M & 132 & 45 & $\mathrm{NC}$ & $\mathrm{NC}$ \\
\hline C009 & 12.42 & M & 112 & 46 & 0 & 42 \\
\hline \multicolumn{7}{|c|}{ Healthy Siblings } \\
\hline S001 & 4.2 & $\mathrm{M}$ & 110 & 42 & 30 & 46 \\
\hline S002 & 6.62 & $\mathrm{~F}$ & NC & NC & NC & NC \\
\hline S003 & 7.47 & M & NC & NC & NC & NC \\
\hline S004 & 7.71 & $\mathrm{~F}$ & 127 & 44 & 1 & 54 \\
\hline S005 & 9.1 & F & 110 & 50 & 6 & 38 \\
\hline S006 & 9.1 & M & 112 & 49 & 9 & 45 \\
\hline S007 & 11.39 & M & 107 & 36 & 6 & 45 \\
\hline S008 & \begin{tabular}{|l|}
12.7 \\
\end{tabular} & F & 118 & 45 & 8 & 52 \\
\hline S009 & \begin{tabular}{|l|l}
13.88 \\
\end{tabular} & F & NC & NC & NC & NC \\
\hline S010 & \begin{tabular}{|l|l|}
14.77 \\
\end{tabular} & F & 112 & 40 & 1 & 24 \\
\hline S011 & \begin{tabular}{|l|l|}
15.89 \\
\end{tabular} & M & 130 & 54 & 12 & 60 \\
\hline \multicolumn{7}{|l|}{ ASD } \\
\hline A001 & 2.96 & M & 78 & 59 & 11 & 54 \\
\hline A002 & 3.63 & M & 65 & 86 & 67 & 73 \\
\hline A003 & \begin{tabular}{|l|}
3.76 \\
\end{tabular} & M & \begin{tabular}{|l|}
77 \\
\end{tabular} & 62 & 28 & 57 \\
\hline A004 & 4.42 & M & 74 & 82 & 21 & 66 \\
\hline A005 & 4.44 & M & 55 & \begin{tabular}{|l|}
87 \\
\end{tabular} & 46 & 67 \\
\hline A006 & \begin{tabular}{|l|l}
4.87 \\
\end{tabular} & M & 72 & 89 & 59 & 74 \\
\hline A007 & \begin{tabular}{|l|l|}
5.02 \\
\end{tabular} & F & 88 & 71 & 50 & 59 \\
\hline A008 & \begin{tabular}{|l}
5.18 \\
\end{tabular} & M & 55 & 86 & 64 & 63 \\
\hline A009 & \begin{tabular}{|l|l|}
5.21 \\
\end{tabular} & M & 66 & 86 & 96 & 76 \\
\hline A010 & 5.44 & M & 65 & 66 & 53 & 59 \\
\hline A011 & 5.6 & M & 55 & 90 & 108 & 77 \\
\hline A012 & 5.62 & M & 61 & 83 & 77 & 83 \\
\hline A013 & 5.66 & M & 63 & 87 & 68 & 78 \\
\hline A014 & 5.68 & $\mathrm{M}$ & 79 & 69 & 5 & 51 \\
\hline A015 & 7.2 & M & 66 & 85 & 83 & 78 \\
\hline A016 & \begin{tabular}{|l}
7.34 \\
\end{tabular} & F & 73 & 76 & 61 & 62 \\
\hline $\mathrm{A} 017$ & 7.44 & M & 66 & 64 & 17 & 51 \\
\hline A018 & 8.06 & F & 59 & 90 & 124 & 75 \\
\hline A019 & 8.28 & M & 108 & 53 & 18 & 69 \\
\hline A020 & 8.38 & M & 53 & 90 & 97 & 76 \\
\hline A021 & 8.65 & M & 87 & 90 & 45 & 57 \\
\hline A022 & 8.72 & M & 48 & 90 & 81 & 64 \\
\hline A023 & 8.82 & M & 78 & 81 & 63 & NC \\
\hline A024 & 8.88 & F & 55 & 90 & 111 & 64 \\
\hline A025 & 8.98 & M & 73 & 88 & \begin{tabular}{|l|}
77 \\
\end{tabular} & 73 \\
\hline A026 & 9.37 & F & 71 & 88 & 49 & 62 \\
\hline A027 & 9.55 & M & 47 & 83 & 58 & 64 \\
\hline A028 & 10.39 & M & 100 & 45 & 25 & 53 \\
\hline A029 & 10.56 & M & 76 & 85 & 56 & 75 \\
\hline $\mathrm{A} 030$ & 10.58 & M & 116 & 70 & 23 & 62 \\
\hline A031 & 11.11 & M & 50 & 82 & 38 & 70 \\
\hline A032 & 11.65 & M & 42 & 85 & 64 & $\begin{array}{l}78 \\
\end{array}$ \\
\hline A033 & 11.96 & M & 62 & 71 & 17 & 54 \\
\hline A034 & \begin{tabular}{|l|l}
13.24 \\
\end{tabular} & M & 80 & 73 & 44 & 60 \\
\hline \multicolumn{7}{|c|}{ Continued } \\
\hline
\end{tabular}




\begin{tabular}{|l|l|l|l|l|l|l|}
\hline Code & Age & Sex & VABS Social & SRS Total & ABC Total & CBCL Total \\
\hline A035 & 13.65 & M & 72 & 81 & 24 & NC \\
\hline A036 & 13.7 & M & 43 & 86 & 39 & 61 \\
\hline A037 & 13.76 & M & 43 & 90 & 80 & 72 \\
\hline A038 & 14.16 & F & 74 & 90 & 81 & 72 \\
\hline A039 & 14.36 & M & 54 & 90 & 121 & 78 \\
\hline A040 & 14.42 & F & 60 & 90 & 64 & NC \\
\hline A041 & 14.49 & M & 57 & 90 & 35 & 65 \\
\hline A042 & 15.35 & M & 46 & 85 & 45 & 69 \\
\hline A043 & 16.42 & F & 58 & 90 & 72 & 74 \\
\hline
\end{tabular}

Table 1. Participant demographics and behavioral scores. Participant demographics along with their VABS, SRS, ABC, and CBCL scores are listed (see "Behavioral Measurements"). NC, not collected.

Our literature search (PubMed search of May 2019) identified 11 completed and published clinical trials (listed in ClinicalTrials.gov) of intranasal oxytocin prescribed for at least 5 days and including at least 10 ASD participants who received oxytocin ${ }^{12,13,15-22}$ (Table 2). Only six of the 11 published studies reported a significant favorable effect of intranasal oxytocin. Notably, of the five studies with a placebo-controlled crossover design, three showed a favorable effect (Table 2). The low number of such clinical trials does not allow reaching a conclusion whether the study design (placebo-controlled crossover, placebo-controlled, or open label) affected the findings. Of note, three out of four trials with children showed favorable outcomes (Table 2) suggesting that intranasal oxytocin might be more likely to be efficacious for children. While findings from these 11 trials are difficult to interpret owing to their variable age groups, dosages, treatment durations and study designs, they clearly indicate that only some ASD individuals may benefit from oxytocin.

Of the 11 trials listed in Table 2, a single trial ${ }^{22}$ examined the OXTR expression levels, and reported lack of correlation of its expression with the effect of intranasal oxytocin on behavioral scores of children diagnosed with ASD. However, this trial measured OXTR expression in whole blood and compared it with the participants Social Responsiveness Scale (SRS) Total Raw Score (while we studied PBMC mRNA and report correlations with ABC scores).

Among the studies listed in Table 2 two studies examined the effects of participants OXTR SNPs on the efficacy of intranasal oxytocin ${ }^{12,13}$; both studies applied a placebo-controlled crossover design in adolescents and adults with ASD. Watanabe et al. ${ }^{12}$ reported that intranasal oxytocin has a smaller effect for participants carrying OXTR rs53576 or rs2254298; while Kosaka et al..$^{13}$ reported lower efficacy for those carrying OXTR rs6791619. Of note, all three of these OXTR SNPs are intronic. We searched for the effects of these OXTR SNPs on its expression in datasets derived from studies with postmortem brain tissues (134 control individuals) deposited in the UK Brain Expression Consortium (UKBEC; http://braineac.org/) by the MRC Sudden Death Brain and Tissue Bank ${ }^{40}$. Our datamining showed that postmortem brain tissues from individuals carrying the minor (A) allele rs53576 allele had significantly higher OXTR expression levels in several brain regions, including frontal cortex, compared with tissues from GG homozygous (Fig. 3). In the general population, rs53576 was associated with general sociality ${ }^{41}$ and empathy ${ }^{42,43}$. Taken together, our current findings on poorer ABC scores in individuals with lower PBMC OXTR expression, along with the findings of the oxytocin clinical trial by Watanabe et al. ${ }^{12,23}$ on better efficacy (improved behavioral scores) in the OXTR rs53576 minor allele carriers, suggest that higher OXTR expression levels may correlate with improved efficacy of intranasal oxytocin in ASD individuals.

Unlike OXTR SNPs, OXTR mRNA levels seem to be more informative of ASD severity: besides DNA methylation and additional epigenetic modifiers of ASD severity ${ }^{44,45}$, they may inform on effects by other non-heritable effectors, such as the gut microbiome, suggested to affect $\mathrm{ASD}^{46,47}$. Based on our findings, we suggest that higher peripheral OXTR expression as detected in PBMCs may reflect differential brain expression and allow improved response to prescribed oxytocin and/or future oxytocin derivatives/agonists. The same considerations may apply also for the prognostic value of higher expression levels of AVPR1A and IGF1 (Fig. 2e-h); while, being supported by the clinical trial findings by Kosaka et al. ${ }^{13}$, the predictive value seems most robust for the PBMC expression of OXTR. Future clinical trials with intra-nasal oxytocin, or future oxytocin derivatives such as oxytocin 5-9 or synthetic agonists ${ }^{48,49}$, should explore the predictive value of the participants' PBMC mRNA of these genes. Findings from such clinical trials with ASD children or adults may allow the future stratification of ASD individuals for the specific therapeutic most likely to benefit them. Once validated by clinical trials, this may allow the future co-marketing of a drug/test combination, along the successful drug/test combinations applied for biologic therapeutics in oncology, such as the Herceptin/Her2 drug/test combination ${ }^{50}$. Considering the increasing rates and high societal cost of ASD, such research efforts seem timely and warranted.

\section{Participants, Materials and Methods}

Participants. The protocol is registered in clinicaltrials.gov as NCT02000284 and was approved by the Institutional Review Board at the University of Arkansas for Medical Sciences (Little Rock, AR). Parents of participants provided written informed consent. All methods were performed in accordance with the relevant guidelines and regulations. Children underwent a fasting blood draw in the morning. Control individuals did not have any neurological disorders or developmental delays. Thus, this study reports gene expression and social and behavioral measurements on a total of 63 children, including 43 children with ASD, 11 non-ASD siblings, and 9 unrelated neurotypical control children. This was a subset of the larger cohort of our study of mitochondrial 

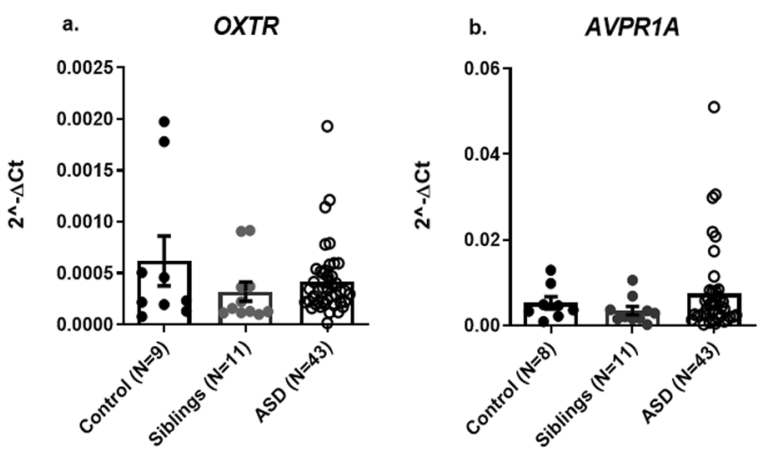

c.
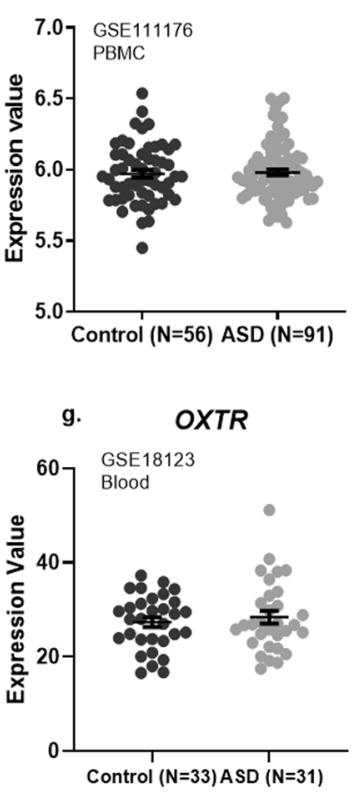

d.

AVPR1A
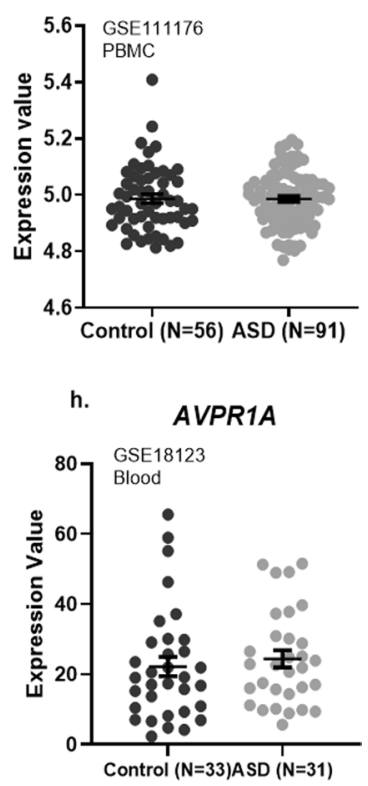
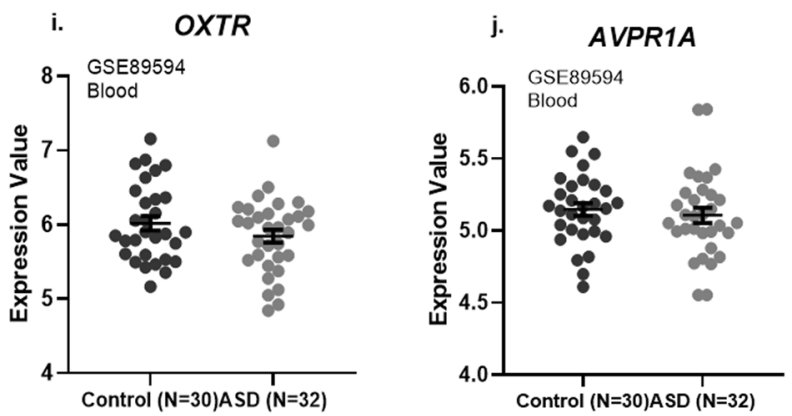

Figure 1. Lack of differences in PBMC mRNA expression levels of OXTR and AVPR1A compared in ASD children, their neurotypical siblings, and neurotypical age-matched children. Findings are shown for our realtime PCR findings $(\mathbf{a}, \mathbf{b})$. Corroborating our findings, no differences between ASD and control children were found in Gene Expression Omnibus (GEO) datasets from PBMC (c-f) or whole blood $(\mathbf{g}-\mathbf{j})$ samples analyzed by datamining for their OXTR and AVPR1A expression. GSE codes and cohort sizes are shown. Note the large distribution of expression levels for OXTR and AVPR1A in both our PBMC samples (a,b) and the GEO datasets $(\mathbf{c}-\mathbf{j})$. Between-group differences were analyzed by one-way ANOVA test $(\mathbf{a}, \mathbf{b})$ and by Student's t-test $(\mathbf{c}-\mathbf{j})$.

function in children with ASD due to limited availability of PBMC for gene expression analysis. Our recent publications outline the methodology for rating participant characteristics of this participant cohort ${ }^{51-53}$.

Behavioral measurements. As mentioned in our previous study ${ }^{53}$, our research staff was trained by a multispecialty team consisting of two licensed psychologists and a speech therapist prior to performing assessments. During the study a research psychologist supervised research staff and provided feedback and retraining if necessary. Observer rated measures included the VABS Survey Interview Form. Parents completed the ABC and the SRS. The VABS is a reliable and valid measure of the ability to perform age-appropriate everyday skills, including communication, daily living, social and motor skills, through a 20-30 minute structured interview with a caretaker ${ }^{54}$. Of note, functional abilities of children with autism are commonly measured with the VABS ${ }^{55}$ as it was in this study. Although some have used IQ to distinguish high and low functioning autism, recent studies indicate that it is a poor predictor ${ }^{56}$. The $\mathrm{ABC}$ is a 58 -item questionnaire ${ }^{53}$ that measures disruptive behaviors and has convergent and divergent validity ${ }^{57}$. The SRS is a 65 -item questionnaire that measures the severity of social skill deficits across five domain ${ }^{58}$ which has been shown to have good correspondence to the gold-standard instrument ${ }^{59}$. The CBCL includes demographic information and ratings of positive behaviors, academic functioning, social competence, and behavior problems commonly applied for classification of behavior disorders ${ }^{60}$.

Blood collection and processing. Samples of $4 \mathrm{ml}$ of venous blood were collected into an ethylenediaminetetraacetic acid-Vacutainer tube, chilled on ice and centrifuged at $1500 \mathrm{~g}$ for 10 minutes at $4{ }^{\circ} \mathrm{C}$ to separate plasma. Plasma was removed and stored at $-80^{\circ} \mathrm{C}$ for later analysis. Plasma was replaced with room temperature wash buffer containing $\mathrm{Ca}^{+2} / \mathrm{Mg}^{+2}$-free PBS with $0.1 \%$ BSA and $2 \mathrm{mM}$ ethylenediaminetetraacetic acid. Diluted 
a.

Vineland Adaptive Behavior Scale

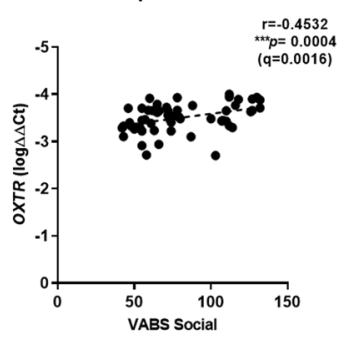

e.

Aberrant Behavior Checklist (Total)

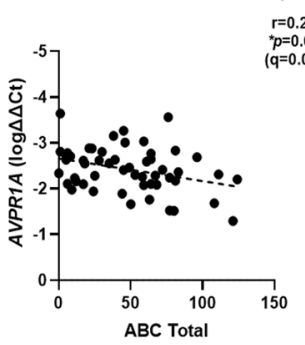

b.

Social Responsiveness Scale (Total)

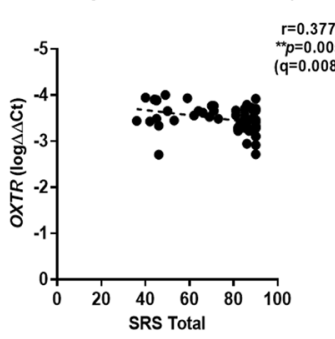

f.

Aberrant Behavior Checklist (Total)

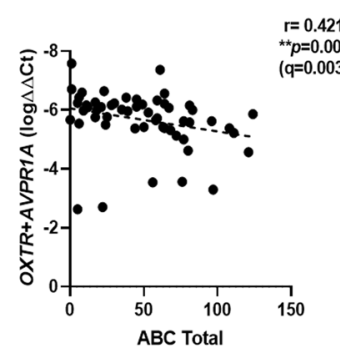

c.

Aberrant Behavior Checklist (Total)

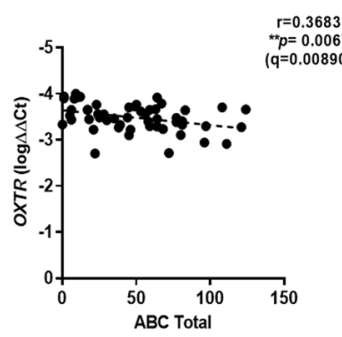

g.

Aberrant Behavior Checklist (Total)

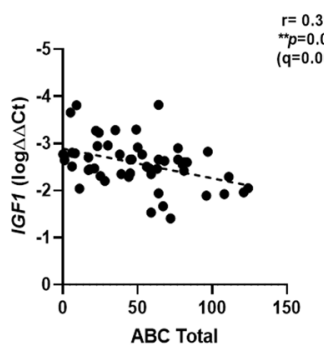

d.

Childhood Behavior Checklist (Total)

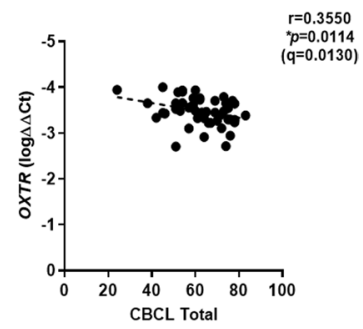

h.

Aberrant Behavior Checklist (Total)

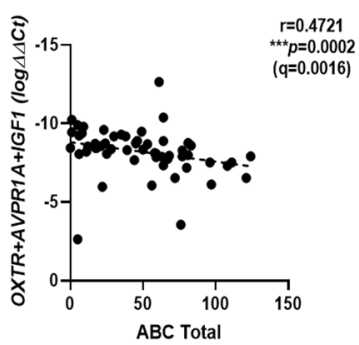

Figure 2. PBMC mRNA expression levels of OXTR, AVPR1A and IGF1 correlate with ASD behavior scores. (a) OXTR vs. VABS scale (social component; $\mathrm{N}=57$ ); (b) OXTR vs. SRS scale $(\mathrm{N}=53$ ); (c) OXTR vs. ABC scale $(\mathrm{N}=53)$; (d) OXTR vs. CBCL scale $(\mathrm{N}=51)$; (e) $A V P R 1 A$ vs. ABC scale $(\mathrm{N}=65)$; (f) sum expression of OXTR + AVPR1A vs. ABC Scale $(\mathrm{N}=56) ;(\mathrm{g}) I G F 1$ vs. ABC scale $(\mathrm{N}=52) ;(\mathbf{h})$ sum expression of OXTR + AVPR1A + IGF1 vs. ABC Scale $(\mathrm{N}=56)$. The $\mathrm{r}$ and $\mathrm{p}$ values for each correlation plot (Spearman test) are shown in each panel. Dotted lines represent the linear regression lines. Note that combining PBMC mRNA expression levels of OXTR and AVPR1A yields more robust correlations compared with each gene separately; while adding PBMC IGF1 mRNA expression levels (as a third gene) further improves the correlation with ABC scores. P values were corrected for multiple testing using Benjamini-Hochberg FDR adjustment (q-values).

\begin{tabular}{|c|c|c|c|c|c|c|c|}
\hline Study & $N^{*}(M / F)$ & Age range & Study design & Dose & Duration & Effect & OXTR genotyped \\
\hline Kosaka $2016^{13}$ & $55(43 / 12)$ & $15-39 y$ & placebo-controlled crossover & 16 or 32 IU/day & 24 weeks* & Yes & rs6791619 \\
\hline Yamasue $2019^{14}$ & $53(53 / 0)$ & $18-48 y$ & placebo-controlled, multi-center & $48 \mathrm{IU} /$ day & 6 weeks & No & No \\
\hline Yatawara $2016^{15}$ & $31(27 / 4)$ & $3-8 y$ & placebo-controlled crossover & 24 IU/day & 14 weeks & Yes & No \\
\hline Higashida $2019^{16}$ & $30(30 / 0)$ & $15-40 y$ & placebo-controlled crossover & $16 \mathrm{IU} /$ day & 8 weeks & No & No \\
\hline Munesue $2016^{17}$ & $29(29 / 0)$ & $15-40 y$ & placebo-controlled crossover & $16 \mathrm{IU} /$ day & 8 weeks & No & No \\
\hline Guastella $2015^{18}$ & $26(26 / 0)$ & $12-18 y$ & placebo-controlled & 36 or $48 \mathrm{IU} /$ day & 8 weeks & No & No \\
\hline Anagnostou $2012^{19}$ & $19(16 / 3)$ & $18-60 y$ & placebo-controlled & $48 \mathrm{IU} /$ day & 6 weeks & Yes & No \\
\hline Dadds $2014^{20}$ & $19(19 / 0)$ & $7-16 y$ & placebo-controlled & 12 or $24 \mathrm{IU} /$ day & 5 days & No & No \\
\hline Watanabe $2015^{12,23}$ & $18(18 / 0)$ & $24-43 y$ & placebo-controlled crossover & $48 \mathrm{IU} /$ day & 6 weeks & Yes & rs53576, rs2254298 \\
\hline Anagnostou $2014^{21}$ & $15(11 / 4)$ & $10-17 y$ & open label (no placebo) & $0.4 \mathrm{IU} / \mathrm{kg} / \mathrm{day}$ & 12 weeks & Yes & No \\
\hline Parker $2017^{22}$ & $14(13 / 1)$ & $6-12 y$ & placebo-controlled & $48 \mathrm{IU} /$ day & 4 weeks & Yes & No \\
\hline
\end{tabular}

Table 2. Published clinical trials with intranasal oxytocin in ASD children and adults. The table summarizes study designs, participant demographics, and outcomes from 11 published placebo-controlled clinical trials with intranasal oxytocin for at least 5 consecutive days in ASD children and adolescents ( 5 trials) or adolescents and adults (6 trials). The list includes trials with PubMed-listed publications that included at least 10 ASD participants who received daily intra-nasal oxytocin and analyzed for the behavioral effects. Trials are listed by decreasing numbers $(\mathrm{N})$ of participants who received oxytocin, completed the study, and were analyzed for behavioral scores prior to and following the study. For trials administering oxytocin twice per day, the total daily dose is shown. In trials showing two doses, the lower dose was assigned for participants with lower weight. *In this trial the 24 week duration includes 4 week washout period between the crossover from oxytocin to placebo or vice versa.

blood was then layered on top of Histopaque-1077 (Sigma Aldrich, St. Louis, MO, USA) and centrifuged at $400 \mathrm{~g}$ for 30 minutes at room temperature. PBMCs were collected, washed twice with wash buffer and counted using a hemocytometer.

RNA isolation. Total RNA was extracted using RNeasy mini kit (Qiagen, Hilden, Germany) from 5 million PBMCs by following manufacturer provided protocol. RNA samples were shipped in 100\% ethanol at room 
a. Frontal cortex

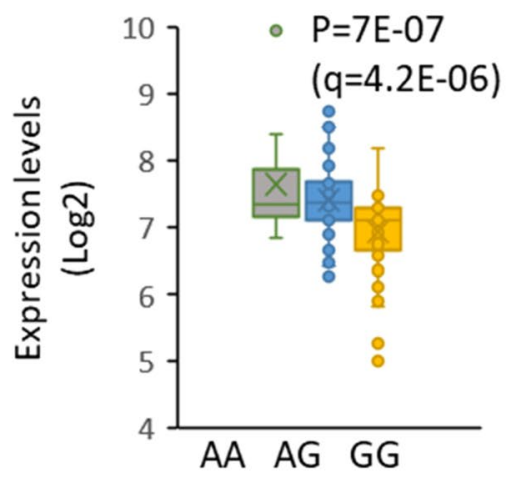

d.

\section{Putamen}

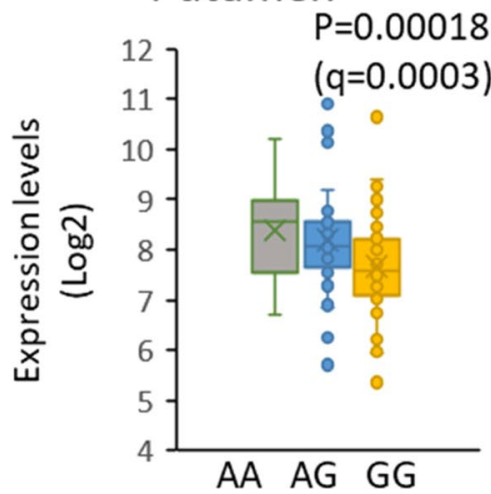

b. Temporal cortex

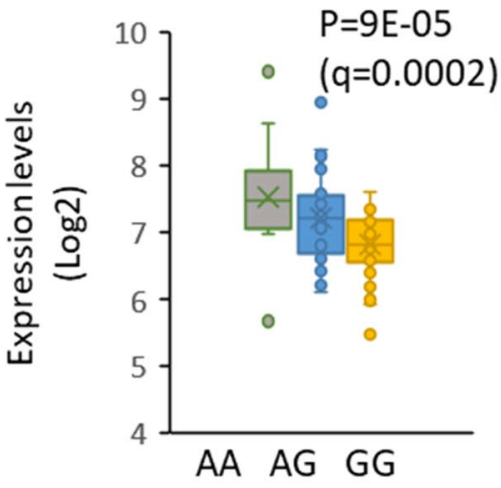

e. Occipital cortex

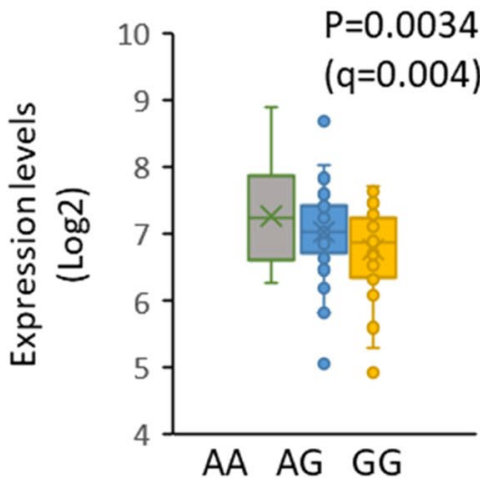

c. Hippocampus

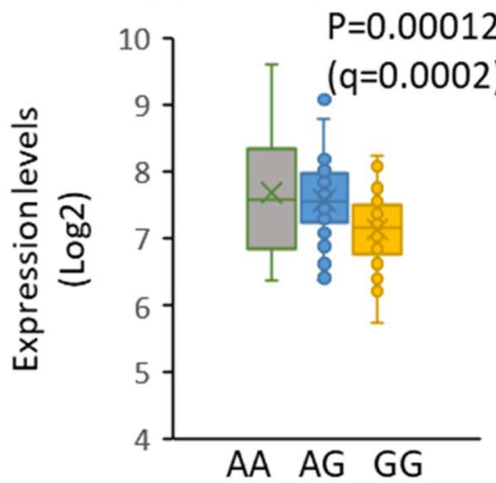

Figure 3. OXTR brain expression levels according to SNP rs53576 alleles (AA, AG, GG). Datamining was performed on the Brain eQTL Almanac website (http://www.braineac.org/). Data were derived from postmortem brain tissues of 111 neuropathologically normal individuals for the following brain regions: (a) frontal cortex; (b) temporal cortex: (c) hippocampus; (d) putamen; (e) occipital cortex (specifically primary visual cortex); and (f) medulla (specifically inferior olivary nucleus). Genotype counts were $A A=10, A G=48$, $\mathrm{GG}=53$. P-values were calculated from the eQTL data by using MatrixEQTL software (according to braineac). $\mathrm{P}$ values were corrected for multiple testing using Benjamini-Hochberg FDR adjustment (q-values).

temperature to Tel-Aviv University. Upon arrival to Tel-Aviv, 10\% 3 M Sodium Acetate and 1ul GlycoBlue ${ }^{\mathrm{TM}}$ (Thermo Fisher Scientific, MA, USA) were added and kept overnight at $-80^{\circ} \mathrm{C}$. Samples were centrifuged at high speed $\left(14,000 \mathrm{~g}, 4^{\circ} \mathrm{C}\right)$ for 30 minutes. Pellets were dried and dissolved in $20 \mathrm{ul}$ DNAse/RNAse-free water.

Real-time PCR. Real-time quantitative PCR (qPCR) reactions were performed with cDNA samples prepared from $1 \mu \mathrm{g}$ RNA samples using qScript cDNA Synthesis Kit (Quanta Bio, MA, USA). Reverse transcription was performed using a thermal cycler over three steps $\left(22^{\circ} \mathrm{C}\right.$ for $5 \mathrm{~min}$, followed by $42^{\circ} \mathrm{C}$ for $30 \mathrm{~min}$ and $85^{\circ} \mathrm{C}$ for $5 \mathrm{~min})$. Real-time PCR reactions were done with $10 \mu \mathrm{l}$ mixtures containing $10 \mathrm{ng}$ of cDNA, PerfeCTa SYBR ${ }^{\circledR}$ Green FastMix Kit (Quanta Bio, MA, USA) and Integrated DNA Technologies, Inc. (Leuven, Belgium) primers (shown below). RPLP0 (Ribosomal Protein Lateral Stalk Subunit P0) was used as reference gene.

\begin{tabular}{|l|l|l|}
\hline Gene & Forward & Reverse \\
\hline OXTR & TCGTGCAGATGTGGAGCGTCT & CATGTAGATCCAGGGGTTGC \\
\hline AVPR1A & TTCTCGTGCCTACGTGACCT & GAGCAGGAACCCCTTTTGGA \\
\hline IGF1 & CAGTTCGTGTGTGGAGACAGGGG & GCAGCACTCATCCACGATGCCT \\
\hline RPLP0 & AGCCCAGAACACTGGTCTC & ACTCAGGATTTCAATGGTGCC \\
\hline
\end{tabular}

GEO datamining. The NCBI Gene Expression Omnibus (GEO) was queried for expression data sets derived from ASD blood or PBMC samples containing cohorts of at least 30 ASD children or adults and a similarly sized age and sex matched control cohort. Data sets from human cell lines, postmortem tissues, and mouse ASD models were excluded. The identified data sets were queried for the expression levels of OXTR and AVPR1A using the GEO2R tool on the NCBI server (https://www.ncbi.nlm.nih.gov/geo/geo2r/). OXTR rs53576 associated brain expression levels were downloaded from the Brain eQTL Almanac website (http://www.braineac.org/). The 
expression levels corresponding to each OXTR rs53576 genotype (AA, AG, and GG) were plotted for six brain regions (Fig. 3). Of note, the above website did not yield information for the OXTR expression associated with rs2254298 or rs6791619 (the two other SNPs mentioned in our Discussion).

Statistical analyses. qPCR data analysis was conducted using the GraphPad Prism v.6 (San Diego, CA, USA). Normality of data distribution was evaluated using the Shapiro-Wilk test; continuous variables between two groups were analyzed by Student's t-test; outliers were detected by Grubbs test. Continuous variables between three groups were analyzed by one-way ANOVA test. P-values $\leq 0.05$ were considered as significant. Data for correlation analysis were log transformed and Spearman correlation test was performed. P-values $\leq 0.01$ were considered significant. P values were adjusted for multiple testing using Benjamini-Hochberg FDR correction ${ }^{61}$. $\mathrm{R}$ statistical software was used to test the robustness of correlations between the sum expression levels of OXTR, $A V P R 1 A$, and IGF1 and the ABC scores (Fig. 2h). A total of 500 random subsets (each containing data from 30 individuals) were independently sampled from our data. For each random sample, the Pearson's correlation coefficient and p-value of the correlation between $\mathrm{ABC}$ score and the summed gene expression was determined. The frequency of correlations with a p-value smaller than 0.05 was then calculated.

\section{References}

1. Jones, C., Barrera, I., Brothers, S., Ring, R. \& Wahlestedt, C. Oxytocin and social functioning. Dialogues Clin Neurosci 19, 193-201 (2017).

2. Maud, C., Ryan, J., McIntosh, J. E. \& Olsson, C. A. The role of oxytocin receptor gene (OXTR) DNA methylation (DNAm) in human social and emotional functioning: a systematic narrative review. BMC psychiatry 18, 154, https://doi.org/10.1186/s12888-018-1740-9 (2018).

3. Gulliver, D. et al. Targeting the Oxytocin System: New Pharmacotherapeutic Approaches. Trends in pharmacological sciences 40, 22-37, https://doi.org/10.1016/j.tips.2018.11.001 (2019).

4. Tully, J., Gabay, A. S., Brown, D., Murphy, D. G. M. \& Blackwood, N. The effect of intranasal oxytocin on neural response to facial emotions in healthy adults as measured by functional MRI: A systematic review. Psychiatry research. Neuroimaging 272, 17-29, https://doi.org/10.1016/j.pscychresns.2017.11.017 (2018).

5. Geng, Y. et al. Oxytocin Facilitates Empathic- and Self-embarrassment Ratings by Attenuating Amygdala and Anterior Insula Responses. Frontiers in endocrinology 9, 572, https://doi.org/10.3389/fendo.2018.00572 (2018).

6. Bartz, J. A., Nitschke, J. P., Krol, S. A. \& Tellier, P. P. Oxytocin Selectively Improves Empathic Accuracy: A Replication in Men and Novel Insights in Women. Biological psychiatry Cognitive neuroscience and neuroimaging, https://doi.org/10.1016/j.bpsc.2019.01.014 (2019).

7. Andari, E., Hurlemann, R. \& Young, L. J. A Precision Medicine Approach to Oxytocin Trials. Curr Top Behav Neurosci 35, 559-590, https://doi.org/10.1007/7854_2017_29(2018).

8. Ruthschilling, C. A. et al. Analysis of transcriptional levels of the oxytocin receptor in different areas of the central nervous system and behaviors in high and low licking rats. Behavioural brain research 228, 176-184, https://doi.org/10.1016/j.bbr.2011.12.005 (2012).

9. Peris, J. et al. Oxytocin receptors are expressed on dopamine and glutamate neurons in the mouse ventral tegmental area that project to nucleus accumbens and other mesolimbic targets. J Comp Neurol 525, 1094-1108, https://doi.org/10.1002/cne.24116 (2017).

10. Freeman, S. M., Inoue, K., Smith, A. L., Goodman, M. M. \& Young, L. J. The neuroanatomical distribution of oxytocin receptor binding and mRNA in the male rhesus macaque (Macaca mulatta). Psychoneuroendocrinology 45, 128-141, https://doi. org/10.1016/j.psyneuen.2014.03.023 (2014).

11. Barrett, C. E., Arambula, S. E. \& Young, L. J. The oxytocin system promotes resilience to the effects of neonatal isolation on adult social attachment in female prairie voles. Translational psychiatry 5, e606, https://doi.org/10.1038/tp.2015.73 (2015).

12. Watanabe, T. et al. Clinical and neural effects of six-week administration of oxytocin on core symptoms of autism. Brain 138, 3400-3412, https://doi.org/10.1093/brain/awv249 (2015).

13. Kosaka, H. et al. Oxytocin efficacy is modulated by dosage and oxytocin receptor genotype in young adults with high-functioning autism: a 24-week randomized clinical trial. Transl Psychiatry 6, e872, https://doi.org/10.1038/tp.2016.152 (2016).

14. Yamasue, H. et al. Effect of intranasal oxytocin on the core social symptoms of autism spectrum disorder: a randomized clinical trial. Molecular psychiatry, https://doi.org/10.1038/s41380-018-0097-2 (2018).

15. Yatawara, C. J., Einfeld, S. L., Hickie, I. B., Davenport, T. A. \& Guastella, A. J. The effect of oxytocin nasal spray on social interaction deficits observed in young children with autism: a randomized clinical crossover trial. Molecular psychiatry 21, 1225-1231, https:// doi.org/10.1038/mp.2015.162 (2016).

16. Higashida, H. et al. Social Interaction Improved by Oxytocin in the Subclass of Autism with Comorbid Intellectual Disabilities. Diseases (Basel, Switzerland) 7, https://doi.org/10.3390/diseases7010024 (2019).

17. Munesue, T. et al. Oxytocin for Male Subjects with Autism Spectrum Disorder and Comorbid Intellectual Disabilities: A Randomized Pilot Study. Front Psychiatry 7, 2, https://doi.org/10.3389/fpsyt.2016.00002 (2016).

18. Guastella, A. J. et al. The effects of a course of intranasal oxytocin on social behaviors in youth diagnosed with autism spectrum disorders: a randomized controlled trial. Journal of child psychology and psychiatry, and allied disciplines 56, 444-452, https://doi. org/10.1111/jcpp.12305 (2015).

19. Anagnostou, E. et al. Intranasal oxytocin versus placebo in the treatment of adults with autism spectrum disorders: a randomized controlled trial. Molecular autism 3, 16, https://doi.org/10.1186/2040-2392-3-16 (2012).

20. Dadds, M. R. et al. Nasal oxytocin for social deficits in childhood autism: a randomized controlled trial. Journal of autism and developmental disorders 44, 521-531, https://doi.org/10.1007/s10803-013-1899-3 (2014).

21. Anagnostou, E. et al. Intranasal oxytocin in the treatment of autism spectrum disorders: a review of literature and early safety and efficacy data in youth. Brain research 1580, 188-198, https://doi.org/10.1016/j.brainres.2014.01.049 (2014).

22. Parker, K. J. et al. Intranasal oxytocin treatment for social deficits and biomarkers of response in children with autism. Proc Natl Acad Sci USA 114, 8119-8124, https://doi.org/10.1073/pnas.1705521114 (2017).

23. Watanabe, T. et al. Oxytocin receptor gene variations predict neural and behavioral response to oxytocin in autism. Soc Cogn Affect Neurosci 12, 496-506, https://doi.org/10.1093/scan/nsw150 (2017).

24. Kim, S. J. et al. Transmission disequilibrium testing of arginine vasopressin receptor 1A (AVPR1A) polymorphisms in autism. Mol Psychiatry 7, 503-507, https://doi.org/10.1038/sj.mp.4001125 (2002).

25. Yirmiya, N. et al. Association between the arginine vasopressin 1a receptor (AVPR1a) gene and autism in a family-based study: mediation by socialization skills. Molecular psychiatry 11, 488-494, https://doi.org/10.1038/sj.mp.4001812 (2006).

26. Uzefovsky, F. et al. Oxytocin receptor and vasopressin receptor 1a genes are respectively associated with emotional and cognitive empathy. Hormones and behavior 67, 60-65, https://doi.org/10.1016/j.yhbeh.2014.11.007 (2015).

27. Johnson, Z. V. \& Young, L. J. Oxytocin and vasopressin neural networks: Implications for social behavioral diversity and translational neuroscience. Neuroscience and biobehavioral reviews 76, 87-98, https://doi.org/10.1016/j.neubiorev.2017.01.034 (2017). 
28. Kolevzon, A. et al. A pilot controlled trial of insulin-like growth factor-1 in children with Phelan-McDermid syndrome. Molecular autism 5, 54, https://doi.org/10.1186/2040-2392-5-54 (2014).

29. Gurwitz, D. Expression profiling: a cost-effective biomarker discovery tool for the personal genome era. Genome medicine 5, 41, https://doi.org/10.1186/gm445 (2013).

30. Liang, L. \& Cookson, W. O. Grasping nettles: cellular heterogeneity and other confounders in epigenome-wide association studies. Human molecular genetics 23, R83-88, https://doi.org/10.1093/hmg/ddu284 (2014).

31. Jin, D. et al. CD38 is critical for social behaviour by regulating oxytocin secretion. Nature 446, 41-45, https://doi.org/10.1038/ nature05526 (2007).

32. Ramaswamy, S. et al. Multiclass cancer diagnosis using tumor gene expression signatures. Proceedings of the National Academy of Sciences of the United States of America 98, 15149-15154, https://doi.org/10.1073/pnas.211566398 (2001).

33. Yukinawa, N. et al. A multi-class predictor based on a probabilistic model: application to gene expression profiling-based diagnosis of thyroid tumors. BMC genomics 7, 190, https://doi.org/10.1186/1471-2164-7-190 (2006).

34. Cizkova, A. et al. Development of a human mitochondrial oligonucleotide microarray (h-MitoArray) and gene expression analysis of fibroblast cell lines from 13 patients with isolated F1Fo ATP synthase deficiency. BMC genomics 9, 38, https://doi. org/10.1186/1471-2164-9-38 (2008).

35. Kwon, J. H. et al. Actionable gene expression-based patient stratification for molecular targeted therapy in hepatocellular carcinoma. PloS one 8, e64260, https://doi.org/10.1371/journal.pone.0064260 (2013).

36. Girard, L. et al. An Expression Signature as an Aid to the Histologic Classification of Non-Small Cell Lung Cancer. Clinical cancer research: an official journal of the American Association for Cancer Research 22, 4880-4889, https://doi.org/10.1158/1078-0432.Ccr$15-2900$ (2016)

37. Young, L. J. Oxytocin and vasopressin as candidate genes for psychiatric disorders: lessons from animal models. American journal of medical genetics 105, 53-54 (2001).

38. Lim, M. M., Bielsky, I. F. \& Young, L. J. Neuropeptides and the social brain: potential rodent models of autism. International journal of developmental neuroscience: the official journal of the International Society for Developmental Neuroscience 23, 235-243, https:// doi.org/10.1016/j.ijdevneu.2004.05.006 (2005).

39. Pobbe, R. L. et al. Oxytocin receptor knockout mice display deficits in the expression of autism-related behaviors. Hormones and behavior 61, 436-444, https://doi.org/10.1016/j.yhbeh.2011.10.010 (2012).

40. Millar, T. et al. Tissue and organ donation for research in forensic pathology: the MRC Sudden Death Brain and Tissue Bank. The Journal of pathology 213, 369-375, https://doi.org/10.1002/path.2247 (2007).

41. Li, J. et al. Association of Oxytocin Receptor Gene (OXTR) rs53576 Polymorphism with Sociality: A Meta-Analysis. PloS one 10, e0131820, https://doi.org/10.1371/journal.pone.0131820 (2015).

42. Gong, P. et al. Revisiting the impact of OXTR rs53576 on empathy: A population-based study and a meta-analysis. Psychoneuroendocrinology 80, 131-136, https://doi.org/10.1016/j.psyneuen.2017.03.005 (2017).

43. Flasbeck, V., Moser, D., Kumsta, R. \& Brune, M. The OXTR Single-Nucleotide Polymorphism rs53576 Moderates the Impact of Childhood Maltreatment on Empathy for Social Pain in Female Participants: Evidence for Differential Susceptibility. Front Psychiatry 9, 359, https://doi.org/10.3389/fpsyt.2018.00359 (2018).

44. Hertz-Picciotto, I., Schmidt, R. J. \& Krakowiak, P. Understanding environmental contributions to autism: Causal concepts and the state of science. Autism research: official journal of the International Society for Autism Research 11, 554-586, https://doi.org/10.1002/ aur.1938 (2018).

45. Wisniowiecka-Kowalnik, B. \& Nowakowska, B. A. Genetics and epigenetics of autism spectrum disorder-current evidence in the field. Journal of applied genetics 60, 37-47, https://doi.org/10.1007/s13353-018-00480-w (2019).

46. Vuong, H. E. \& Hsiao, E. Y. Emerging Roles for the Gut Microbiome in Autism Spectrum Disorder. Biological psychiatry 81, 411-423, https://doi.org/10.1016/j.biopsych.2016.08.024 (2017).

47. Pulikkan, J., Mazumder, A. \& Grace, T. Role of the Gut Microbiome in Autism Spectrum Disorders. Advances in experimental medicine and biology 1118, 253-269, https://doi.org/10.1007/978-3-030-05542-4_13 (2019).

48. Moy, S. S. et al. Prosocial effects of an oxytocin metabolite, but not synthetic oxytocin receptor agonists, in a mouse model of autism. Neuropharmacology 144, 301-311, https://doi.org/10.1016/j.neuropharm.2018.10.036 (2019).

49. Ichinose, W. et al. Development of a Highly Potent Analogue and a Long-Acting Analogue of Oxytocin for the Treatment of Social Impairment-Like Behaviors. Journal of medicinal chemistry 62, 3297-3310, https://doi.org/10.1021/acs.jmedchem.8b01691 (2019).

50. Albrecht, H. Trastuzumab (Herceptin(R)): overcoming resistance in HER2-overexpressing breast cancer models. Immunotherapy 2 , 795-798, https://doi.org/10.2217/imt.10.71 (2010).

51. Delhey, L. et al. Bioenergetic variation is related to autism symptomatology. Metabolic brain disease 32, 2021-2031, https://doi. org/10.1007/s11011-017-0087-0 (2017).

52. Delhey, L. M. et al. The Effect of Mitochondrial Supplements on Mitochondrial Activity in Children with Autism Spectrum Disorder. Journal of clinical medicine 6, https://doi.org/10.3390/jcm6020018 (2017).

53. Frye, R. E. et al. Folinic acid improves verbal communication in children with autism and language impairment: a randomized double-blind placebo-controlled trial. Molecular psychiatry 23, 247-256, https://doi.org/10.1038/mp.2016.168 (2018).

54. Frye, R. E. et al. Metabolic effects of sapropterin treatment in autism spectrum disorder: a preliminary study. Translational psychiatry 3, e237, https://doi.org/10.1038/tp.2013.14 (2013).

55. Szatmari, P. et al. Developmental trajectories of symptom severity and adaptive functioning in an inception cohort of preschool children with autism spectrum disorder. JAMA Psychiatry 72(3), 276-83 (2015).

56. Alvares, G. A. et al. The misnomer of 'high functioning autism': Intelligence is an imprecise predictor of functional abilities at diagnosis. Autism, https://doi.org/10.1177/1362361319852831 (2019).

57. Kaat, A. J., Lecavalier, L. \& Aman, M. G. Validity of the aberrant behavior checklist in children with autism spectrum disorder. Journal of autism and developmental disorders 44, 1103-1116, https://doi.org/10.1007/s10803-013-1970-0 (2014).

58. Constantino, J. N. In Encyclopedia of Autism Spectrum Disorders (ed Fred R. Volkmar) 2919-2929 (Springer New York, 2013).

59. Murray, M. J., Mayes, S. D. \& Smith, L. A. Brief report: excellent agreement between two brief autism scales (Checklist for Autism Spectrum Disorder and Social Responsiveness Scale) completed independently by parents and the Autism Diagnostic InterviewRevised. Journal of autism and developmental disorders 41, 1586-1590, https://doi.org/10.1007/s10803-011-1178-0 (2011).

60. Nolan, T. M. et al. Child Behaviour Checklist classification of behaviour disorder. Journal of paediatrics and child health 32, 405-411 (1996).

61. Benjamini, Y. \& Hochberg, Y. Controlling the False Discovery Rate: A Practical and Powerful Approach to Multiple Testing. Journal of the Royal Statistical Society Series B (Methodological) 57, 289-300 (1995).

\section{Acknowledgements}

R.E.F. and S.R. were supported by the Arkansas Biosciences Institute (Little Rock, AR, USA), The Jonty Foundation (St Paul, MN), The Autism Research Institute (San Diego, CA), the Gupta Family Foundation (Atherton, CA), The Jane Bostford Johnson Foundation (New York, NY), and the Jager Family Foundation (Chicago, IL). D.G. was supported by the Yoran Institute for Human Genome Research at Tel Aviv University (Tel Aviv, Israel). The authors thank Adva Hadar for assistance with bioinformatics datamining, Dr. Ran Elkon 
for help with statistics analysis, and David Groenewoud for help with testing the robustness of correlations of summed gene expression levels with $\mathrm{ABC}$ scores using R software.

\section{Author Contributions}

D.G., S.R. and R.E.F. conceived the study and wrote the manuscript. R.E.F. and S.R. were the Principal Investigators for NCT02000284 which was the study which collected that behavior, cognitive and biological data. S.B. separated PBMCs from fresh blood samples and extracted their RNAs. I.V. prepared the cDNAs, conducted the real-time PCR experiments, analysed the results, and prepared the figures and tables. J.S. assisted with realtime PCR experiments. All authors reviewed the manuscript.

\section{Additional Information}

Supplementary information accompanies this paper at https://doi.org/10.1038/s41598-019-49617-9.

Competing Interests: The authors declare no competing interests.

Publisher's note: Springer Nature remains neutral with regard to jurisdictional claims in published maps and institutional affiliations.

(c) (i) Open Access This article is licensed under a Creative Commons Attribution 4.0 International License, which permits use, sharing, adaptation, distribution and reproduction in any medium or format, as long as you give appropriate credit to the original author(s) and the source, provide a link to the Creative Commons license, and indicate if changes were made. The images or other third party material in this article are included in the article's Creative Commons license, unless indicated otherwise in a credit line to the material. If material is not included in the article's Creative Commons license and your intended use is not permitted by statutory regulation or exceeds the permitted use, you will need to obtain permission directly from the copyright holder. To view a copy of this license, visit http://creativecommons.org/licenses/by/4.0/.

(C) The Author(s) 2019 